\title{
A Task Recommendation system for children and youth with Autism Spectrum Disorder
}

\author{
Margarida Costa ${ }^{1}$, Angelo Costa ${ }^{1}$, Vicente $\mathrm{Julian}^{2}$, and Paulo Novais ${ }^{1}$ \\ 1 Centro ALGORITMI, Escola de Engenharia, Universidade do Minho, Braga, \\ Portugal \\ margaridafsc@gmail.com, \{acosta, pjon\}@di.uminho.pt \\ 2 Departamento de Sistemas Informáticos y Computación, Universitat Politècnica de \\ València, Spain \\ vinglada@dsic.upv.es
}

\begin{abstract}
Current studies indicate that 1 in 68 children have Autism Spectrum Disease. It is known that early diagnosis and intervention can alter the course of development and significantly improve the prognosis of the disease. It is our intention to develop a task Recommendation System, which will use a Case-based Reasoning machine learning technique, in order to supplement the child's regular therapy. Besides the tasks' recommendation, this application will allow a closer monitoring by parents and a better coordination with the therapists, contributing to improve the results on child's development.
\end{abstract}

Keywords: Autism Spectrum Disease, Case-based Reasoning, Decision Support System, Mobile Computing

\section{Introduction}

Autism Spectrum Disease (ASD) is part of a group of complex disorders in brain neurodevelopmental disorders [1]. This disorder is mainly characterized by verbal and non-verbal communication problems; difficulties in relating to people, things, and events; and repetitive body movements or behaviors [1]. Although those are defined criteria by the Diagnostic and Statistical Manual of Mental Disorders (DSM-V), autism differs from person to person, in severity and combinations of symptoms and there is not a medical test that can diagnose it [2].

In 2014, the CDC (United States) has released a study that found that 1 in 68 children aged 8 years had ASD [3]. In Portugal, the prevalence of ASD in 2007 estimated 10 per 10000 children [4].

Although there is still no cure for autism, is known that early diagnosis and intervention can alter the course of children development. That is why therapy and a regular training and support from parents can significantly improve the prognosis of the disease [2]. Children with autism often need external stimulus to initiate, maintain, or terminate an activity [5].

Technological advancements gave devices the ability to deliver visual, tactile and mechanic feedback, which can be used for children with autism, since this kind of stimulus are generally better received than human stimulus [5]. 
Combining the technology advantages with the necessities of an autistic child, the motivation for developing this application arose. This paper presents a prototype of our idea. The next section presented the related work. In section 3 is presented the relevant state of the art. Section 4 makes a brief description of the application architecture. The visual interfaces are described in section 5. Section 6 is dedicated to the CBR and the Recommendation System. The last section is dedicated to the conclusions and future work.

\section{Related Work}

Published in 1992, by Boston University, the Pediatric Evaluation of Disability Inventory (PEDI) was a paper-pencil survey, which asked the caregivers of children and youth with disabilities about their difficulty in doing an extensive list of activities [6]. Later, a Computer Adaptive Test (PEDI-CAT), has been created to increase efficiency and reduce the respondent burden. This survey enables clinicians to construct a description about the current functional status or progress in acquiring functional skills for children between 1 and 21 years of age [6]. The PEDI-CAT has a "item bank" that is an extensive list of 276 activities in the domains of Daily Activities, Social/Cognitive, Responsibility and Mobility [6].

After the completion of one PEDI-CAT, a score report is available for each domain administered, which includes three distinct types of scores: the scaled scores, the normative scores and the item-maps. The scaled scores are the result of the caregivers responses to the questioned items, calculated through a statistical model. The item maps are tables (one for each domain), that define the expected level of difficulty/responsibility for each activity, based on the child's obtained scaled score [6].

PEDI-CAT-ASD, is an adaptation of PEDI-CAT for children and youth with autism spectrum disorders. The results have shown that PEDI-CAT-ASD is appropriate to assess children and youth with ASD among an heterogeneous range of age and severity [7].

\section{State of the Art}

Currently, there are a huge variety of applications in this area, which can be categorized according to their target audience: if is for children with ASD or their caregivers; their main propose: learning skills or logistic and organizational issues; and the domain of learning skills to explore. Regarding applications directed to the education of children with ASD we present some examples available in the market:

MITA was developed by a group composed by a neuroscientist, an earlychild-development specialist, artists, developers and therapists. Mental Imagery Therapy for Autism (MITA) is based on Pivotal Response Treatment which have as target three critical areas of development: response to multiple cues, motivation and self-management. This application includes nine adaptive games 
that, over time, get more difficult and aim to improve the child's development specifically in the areas of language, attention and visual skills [8].

Social Skills for Autism has been developed by the Shine Centre for Autism, in Ireland, and is based on the Personal \& Life Skills Programme (PALS) [9]. This application has 17 individual lesson plans, each one covering a required social skill. When children complete one plan it gives them a reward [10].

Choiceworks aims to help caregivers provide their children clear and easy rules and timetables which allow them to be more independent and to adapt their emotions and behaviors according to different situations. This application is provided with three customizable boards and a multimedia library. It allows the creation of profiles, managing multiple users and saving boards. It can also be adapted for the usage of teachers in a school environment [11].

Autism Tracker by Track \& Share Apps company, allows users to explore autism, tracking and analyzing a large collection of data such as sleep, stress, sensory arousal, happiness, activity level and behavior. It has a visual calendar that can summarize totals, averages and statistics over a select period of time, as well, scheduling activities. It also give the possibility to share data with the other users through social networks [12].

Pathfinder is the result of the merge of specialization in strategies for simplification of behavioral data and health-care and autism therapy practice. It was specifically developed to provide technology solutions for administrative and data collection issues, for behavioral health providers. It has easy interfaces for the data store and the creation of documents and shows the therapies progress. Besides that, it organizes therapy teams and schedule and notify therapists about their work day [13].

\section{Architecture}

The main goal of this project is to improve the interaction methods through technology, thus improving the results of therapy $[14,15]$.

A client-server system have been developed: an Android Application that aims to improve the communication between therapists, children and parents, as well as help the children doing their tasks in a fun way; and a Recommendation System for suggesting activities that are appropriate, necessary and enjoyable for each children, using Case-Based Reasoning (CBR) [16, 17].

We have based our data on the PEDI-CAT-ASD repository. The autistic sample includes the scaled scores, per domain, for the ages of 5,10 and 15 years, obtained through a questionnaire made to 105 parents of autistic children.

The system described in Figure 1 operates in the following way:

1. The user Parent introduces the age of his child in the system;

2. Based on the inserted age, the system selects the predicted Scaled Score in each one of the domains, according to the autistic sample;

3. The difficulty/responsibility level for each incorporated activity in the system is given, according to the previously obtained Scaled Score and the item-map;

4. The user Therapist introduces the difficulty level in the system; 


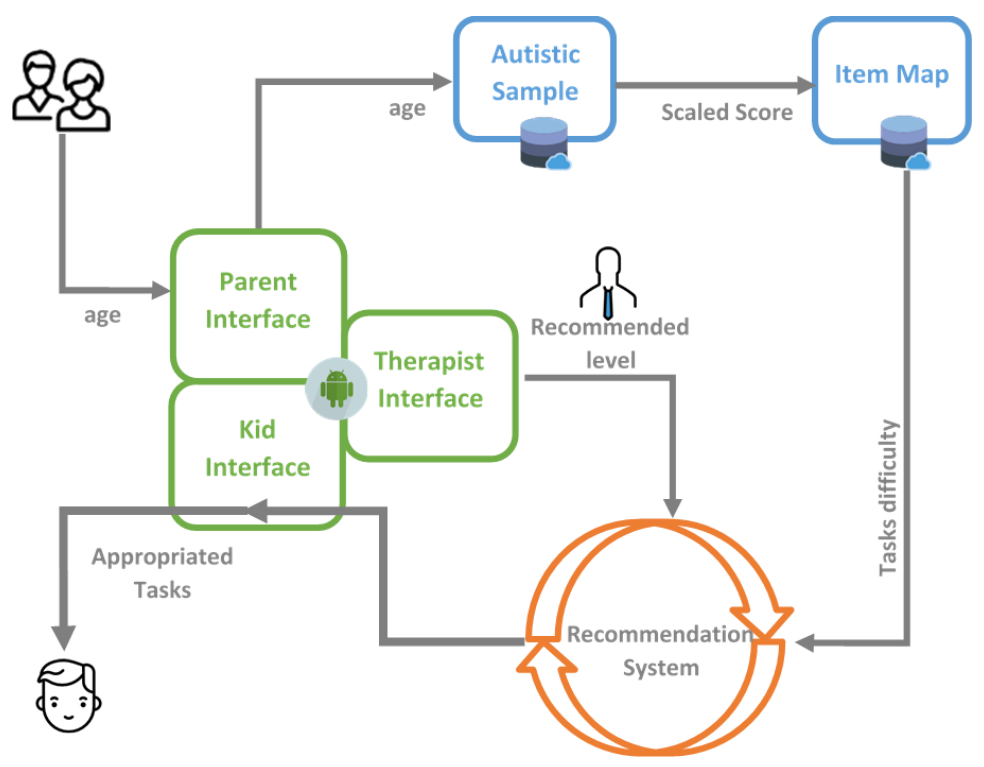

Fig. 1. Client-server system architecture.

5. With the previous information, and other external data, the Recommendation System is fed and is ready to start operating. In section 6 it is explained in detail;

6. The user Kid receives the assigned tasks.

\section{$5 \quad$ Visual Interfaces}

The mobile application has three profiles, as seen in Fig. 2 a: Parent, Kid and Therapist. The Kid account is authored by the parents and require the children data and one of the therapists available in the system (Fig. 2 c).

After login, parents and therapists are received with a list of their children. The Figure (3 a) shows the main page for Kid and for his Parent and Therapist (b). The Kid profile has tasks and rewards in an effort of increase the interaction with the system. In the Tasks interface, children have a list of suggested activities, which they can select and mark as done or undone. When a task is marked as "done" it is removed from that list and, pending the parents approval, is moved to the "Tasks to approve" list on the parents interface. Each task has a symbolic value which is given according to the activity difficulty. After the parent's approval, the child receives that value. The main interface for parents and therapists has the following options: Tasks, Rewards, Diary and Analytics. In Tasks the parents are able to propose new tasks for their children, either automatically chosen by the Recommendation System, or manually chosen from the item bank. It has two separated lists of tasks: "Tasks to do" and "Tasks to approve" (Fig. 4 a); the second list marks the "done" and "undone" tasks. In 

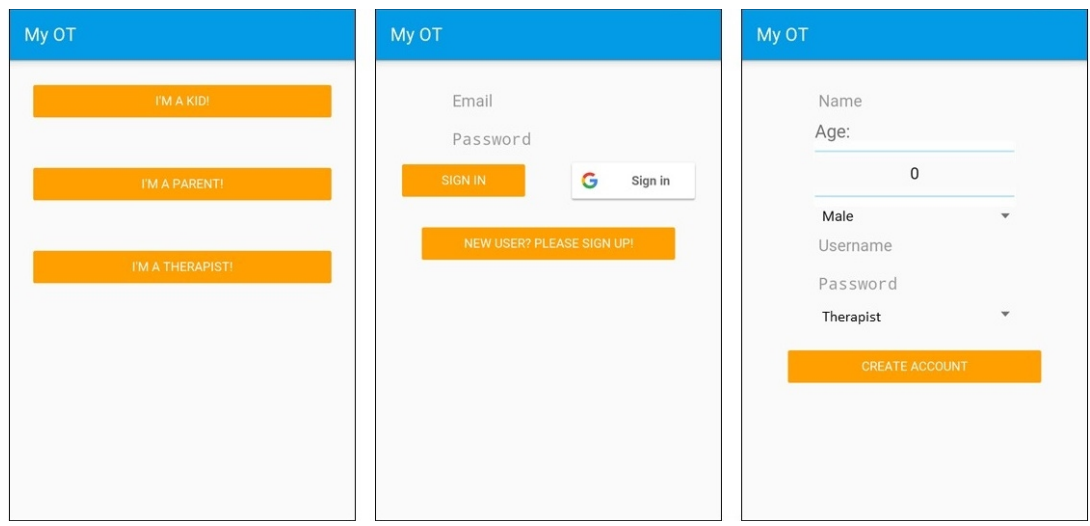

Fig. 2. (a) Choose type of user, (b) login and (c) Create kids account interfaces.

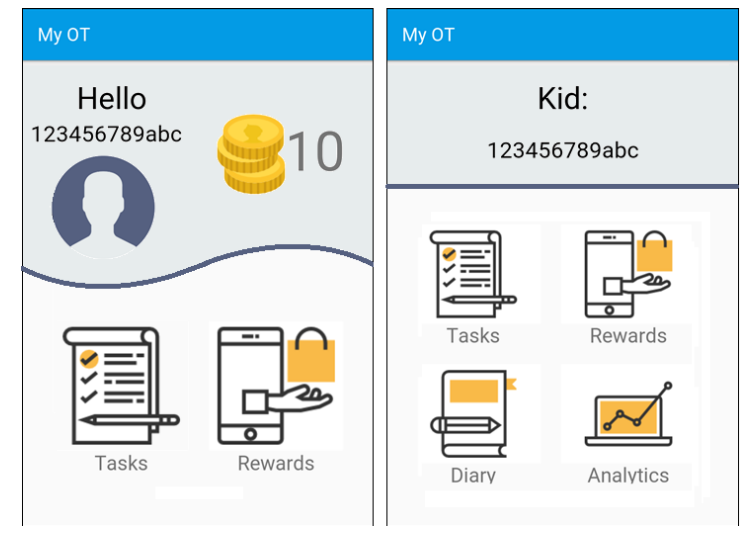

Fig. 3. (a) Kids Application mains page; (b) Parents/Therapists main page

case of "done" the parent can classify the difficulty on doing that activity (Fig. 4 b). The therapists' interface for Tasks is similar, but without buttons to propose new tasks or the classification option. Therapists have access to the tasks lists, to follow the work done by the patient. In the Rewards interface, parents and therapists can add new prizes to the rewards list. The Diary button, opens a log book, where therapists may register the most important and worked goals of each session. Thus, parents are able to see their child's progress.

The Analytics interface for parents and therapists is able to show a domain that the user wants to analyse. The statistics show the frequency of each difficulty level for the selected domain.

\section{CBR Methods}

To provide more accurate suggestions a CBR system was adopted [18]. 

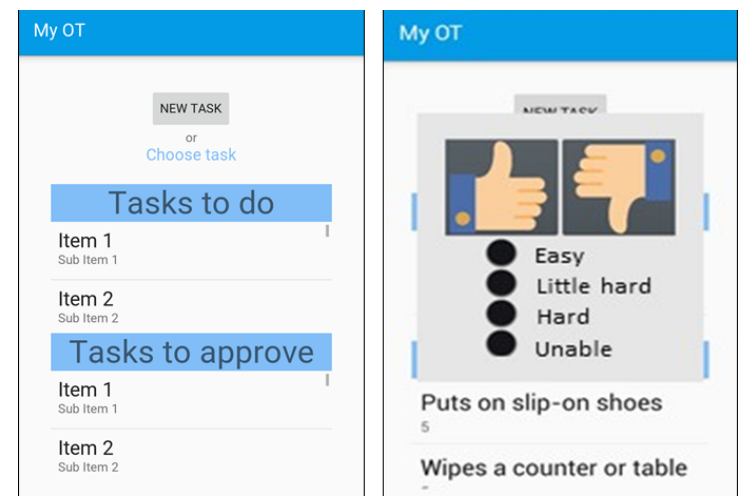

Fig. 4. Tasks activity interface.

Following this approach we have defined first the set of attributes that form our proposed case. In this platform our problem is described by the age of the child, the gender, the day time (morning/afternoon), the week time (week/weekend), the therapist ID and the level of difficulty; and the solution is represented by one task ID. In some cases, some attributes may have missing values, but we foresee that the system should be able to handle incomplete information.

Table 1. A Case base example.

\begin{tabular}{|c|c|c|c|c|}
\hline \multicolumn{4}{|c|}{ Description } & Solution \\
\hline caseID age & gender dayTime weekTime & therapistID & difficulty & taskID \\
\hline $\mathrm{c} 00015$ & 1 & thera@gmail.com & 4 & DA001 \\
\hline
\end{tabular}

In table 1, an example for the case c0001 is shown. The gender, dayTime and weekTime are binary attributes. In gender, 0 represents "female" and 1 "male"; In dayTime, 0 means "afternoon" and 1 "morning" and in weekTime, 0 represents "weekend" and 1 "working days".

The first phase of the cycle - the retrieval phase - retrieves similar cases from the stored ones. For the retrieval algorithm, the most similar cases are selected by means of a k-nearest neighbour algorithm using the local similarity measures to compute the similarity among cases.

For the age attribute a threshold function has been applied.

The value of the threshold was defined as 2 , in order to cover all ages in the autistic sample.

For the difficulty attribute, an interval function. This function returns $1-\mid$ difficultynew - difficultycaseBase $\mid / k$, where $k$ is 4 , the number of stages in our difficulty scale. In the rest of the attributes an equal function was used. 
The global similarity is calculated through a weighted average function with the values of the local similarity and the respective weights of the attributes. In consensus with a team of occupational therapists, we have decided to give more importance to the age and difficulty attributes, weighing 1 , while the other attributes receive a 0,5 weight. The global similarity retrieves a value between 0 and 1 , where 1 means a perfect match.

After a solution has been applied, the user must indicate to the system whether that solution is optimal to the problem or not, which is done when parents confirm that his child has done the task and its difficulty. Each time that one solution is used, its suitability degree is increased and the difficulty value is updated averaging the old and new values. That is how the system learns the degree of suitability of the solutions that the CBR engine provides, by means of the revision and retention phases. Finally, if the similarity of the stored cases is below the acceptance threshold, the system selects the most similar solution and saves it as a new case, gathering the acceptance information in the next cycle.

\section{Conclusions \& Future Work}

This work presents an initial implementation of a therapy support system for children and youth with ASD. Our aim is to produce a companion system that is used as a reinforcement for the therapy sessions that the children attend. We believe that our system will increase the interaction and responsiveness of all users involved, thus optimizing the therapy procedures, and give the opportunity to parents and clinicians to monitoring their children more closely. Comparing with other solutions available in the market, our application is very complete once it provides skill learning trough an automated system, and also data collecting and analysis for caregivers.

Our team of occupational therapists indicate that this application may improve the results of therapy, although validation with real users is needed to confirm these allegations. Also, after conducting a survey on the usability of this application, we have data indicating that the idea is being very well received by health and education professionals dealing with autistic children.

As future work, in order to make our Recommendation System more efficient, it would be important to improve the quality of the cases in the CBR. We also want to adapt the language of the activities to a more appropriated one for children and to illustrate every possible activity. Finally, we want to test

our application in a representative sample of children and youth with ASD, in Portugal.

\section{Acknowledgements}

This work is partially supported by the MINECO/FEDER TIN2015-65515-C4-1-R. This work is supported by COMPETE: POCI-01-0145-FEDER-007043 and FCT Fundação para a Ciência e Tecnologia within the projects UID/CEC/00319/ 2013 and Post-Doc scholarship SFRH/BPD/102696/2014 (A. Costa). 


\section{References}

1. American Psychiatric Association: Diagnostic and statistical manual of mental disorders. 5th edn. Arlington, VA: American Psychiatric Publishing (2013)

2. American Psychiatric Association: What Is Autism Spectrum Disorder? https://goo.gl/pKX8IZ (2016)

3. Christensen, D.L., Baio, J., Braun, K.V.N., et al.: Prevalence and Characteristics of Autism Spectrum Disorder Among Children Aged 8 Years - Autism and Developmental Disabilities Monitoring Network, 11 Sites, United States, 2012. Morbidity and mortality weekly report. Surveillance summaries (Washington, D.C. : 2002) 65(3) (2014) 1-23

4. Oliveira, G., Ataíde, A., Marques, C., et al.: Epidemiology of autism spectrum disorder in Portugal: prevalence, clinical characterization, and medical conditions. Developmental Medicine \& Child Neurology 49(10) (2007) 726-733

5. Goldsmith, T., LeBlanc, L.: Use of Technology in Interventions for Children with Autism. Journal of Early and Intensive Behavior 1(2) (2004) 166-178

6. Haley, S.M., Coster, W.J., Dumas, H.M., et al.: PEDI-CAT version 1.3.6: Development, standardization and administration manual. Health and Disability Research Institute, Boston University School of Public Health, Boston University Medical Center, Boston, MA Funded (2012)

7. Coster, W.J.., Kramer, J.M., Tian, F., et al.: Evaluating the appropriateness of a new computer-administered measure of adaptive function for children and youth with autism spectrum disorders. Autism 20(1) (2016) 14-45

8. Google Play: Autism Therapy with MITA. https://goo.gl/PPxgVN

9. The Shine Centre: Personal and Life Skills Programme. http://shineireland.com/pals/

10. Google Play: Social Skills for Autism. https://goo.gl/f6WA3N

11. Bee Visual: Choice Works. http://www.beevisual.com/

12. TracknShare: Autism Tracker. http://tracknshareapp.com/autism-tracker/

13. Pathfinder Health Innovations: All-In-One Therapy Solutions. https://pathfinderhi.com/

14. Chen, Q., Yan, Z.: Does multitasking with mobile phones affect learning? A review. Computers in Human Behavior 54 (2016) 34-42

15. Redmayne, M., Smith, C.L., Benke, G., et al.: Use of mobile and cordless phones and cognition in Australian primary school children: a prospective cohort study. Environmental Health 15(1) (2016) 26

16. Costa, A., Julián, V., Novais, P.: Advances and trends for the development of ambient-assisted living platforms. Expert Systems 00(00) (2016)

17. Saraiva, R., Perkusich, M., Silva, L., et al.: Early diagnosis of gastrointestinal cancer by using case-based and rule-based reasoning. Expert Systems with Applications 61 (2016) 192-202

18. Aamodt, A.: Case-Based Reasoning : Foundational Issues, Methodological Variations, and System Approaches. 7 (1994) 39-59 